\title{
Kaluza-Klein Reduction of a Quadratic Curvature Model
}

\author{
S. Başkal 1 and H. Kuyrukcu 2 \\ Physics Department, Middle East Technical University \\ 06531, Ankara, Turkey
}

\begin{abstract}
Palatini variational principle is implemented on a five dimensional quadratic curvature gravity model, rendering two sets of equations which can be interpreted as the field equations and the stress-energy tensor. Unification of gravity with electromagnetism and the scalar dilaton field is achieved through the Kaluza-Klein dimensional reduction mechanism. The reduced curvature invariant, field equations and the stress-energy tensor in four dimensional spacetime are obtained. The structure of the interactions among the constituent fields is exhibited in detail. It is shown that the Lorentz force naturally emerges from the reduced field equations and the equations of the standard Kaluza-Klein theory is demonstrated to be intrinsically contained in this model.
\end{abstract}

\section{Introduction}

Theories in dimensions higher than four seem to be promising candidates for the ultimate unification of fundamental forces. The almost ninety years old Kaluza-Klein (KK) theory unifying electromagnetism with gravity in 5D [1], currently appears as a part of more involved models [2, 3], may still have some aspects that deserve to be investigated in their own right. As is well known, the standard KK (SKK) theory is obtained by the metric variations of the 5D Einstein-Hilbert $(\mathrm{EH})$ action [4. Since then there have been many alternative approaches to the theory dealing with various types of actions [5], including those that contain dimensionally extended Euler densities [6, 7, 8, 9, 10]. Usually, the field equations are obtained by metric variations of the action. In this work, we shall deviate from this common practice and employ the Palatini variational principle which takes variations

\footnotetext{
${ }^{1}$ electronic address: baskal@newton.physics.metu.edu.tr

2 electronic address: halil@metu.edu.tr
} 
both with respect to the connection and to the metric by treating them as independent variables [11].

If the requirement on the order of the derivatives of the metric in the equations is released, then we can proceed with the simplest gravity model and consider an action which is quadratic in the curvature. Motivations for choosing such an action is more substantial than seeking for simplicity or pursuing for analogy with other gauge theories whose field equations and the stress-energy (SE) tensors are derived from an action quadratic in their fields. In addition to its natural emergence as the leading term in string generated gravity models in their low energy limit [12, when coupled to matter its renormalizability problems become much less severe 13 .

Here, we shall investigate the consequences of the KK reduction mechanism on the 5D field equations and the SE tensor obtained from implementing the Palatini variational principle to a quadratic curvature 5D gravity model.

\section{The quadratic curvature model in five dimen- sions}

The basic operating mechanism of the KK theory can safely be viewed as a spontaneous compactification of the five dimensional spacetime $M_{5}$ (with coordinates $\left.\left(x^{a}, y\right)\right)$ to $M_{4} \times S^{1}$ while the $5 \mathrm{D}$ Poincaré symmetry $P_{4}$ of $M_{5}$ is spontaneously broken to $P_{5} \times U(1)$. Here, $M_{4}$ is the actual spacetime and $S^{1}$ has the topology of a circle whose radius is assumed to be at the order of the Plank length. The line element on $M_{5}$ is written as

$$
d s_{5}^{2}=\hat{g}_{A B} \hat{e}^{A} \otimes \hat{e}^{B},
$$

with its signature adopted as $(-,+,+,+,+)$. The capital indices $A, B, \ldots$ assume the values $0,1,2,3,5$ and the lower case indices $j, k, \ldots$ run from 0 to 3 . We shall be working in the horizontal lift basis (HLB) which will prove to be convenient for our purposes. An adequate amount of detail in the context of the SKK theory coupled to the Dirac field can be found in [14. In a more general framework it is referred as an anholonomic basis and elucidated in [15.

Then the metric $\hat{g}_{A B}$ takes the form

$$
\left(\begin{array}{cc}
g_{i j} & 0 \\
0 & 1
\end{array}\right)
$$


with the basis

$$
\hat{e}^{j}\left(x^{a}, y\right)=d x^{j}, \quad \hat{e}^{5}\left(x^{a}, y\right)=\varphi\left(x^{a}\right)\left(d y+A_{k}\left(x^{a}\right) d x^{k}\right) .
$$

The SKK theory uses the 5D EH action

$$
\hat{S}_{E H}=\int(-\hat{g})^{\frac{1}{2}} \hat{R} d^{5} x
$$

where $\hat{R}$ is the $5 \mathrm{D}$ curvature scalar. The field equations of the theory $\hat{R}_{A B}=0$, are obtained through metric variations. Using the KK reduction mechanism they are expressed as [16]:

$$
\begin{aligned}
& K 1_{a b} \equiv R_{a b}-\frac{1}{2} \varphi^{2} F_{a k} F_{b}{ }^{k}+\varphi^{-1} D_{a} \varphi_{b}=0 \\
& K 2_{k} \equiv D_{j} F^{j}{ }_{k}+3 \varphi^{-1} \varphi^{j} F_{j k}=0 \\
& K 3 \equiv D_{j} \varphi^{j}-\frac{1}{4} \varphi^{3} F_{j k} F^{j k}=0 .
\end{aligned}
$$

Here, $R_{a b}$ is the $4 \mathrm{D}$ Ricci tensor, $F_{a b}=\partial_{a} A_{b}-\partial_{b} A_{a}$ is the electromagnetic (EM) field strength tensor and $\varphi$ is the dilaton field. For convenience, we also give the reduced forms of the 5D curvature tensor in HLB:

$$
\begin{aligned}
& \hat{R}_{a b m n}=R_{a b m n}-\frac{1}{4} \varphi^{2}\left(2 F_{a b} F_{m n}+F_{a m} F_{b n}-F_{a n} F_{b m}\right) \\
& \hat{R}_{5 b m n}=\frac{1}{2} \varphi D_{b} F_{m n}+\frac{1}{2}\left(2 \varphi_{b} F_{m n}+\varphi_{m} F_{b n}-\varphi_{n} F_{b m}\right) \\
& \hat{R}_{a 5 m 5}=-\varphi^{-1} D_{m} \varphi_{a}-\frac{1}{4} \varphi^{2} F_{a j} F_{m}^{j} .
\end{aligned}
$$

In this article, we shall consider an alternative 5D action

$$
\hat{S}=\int \mathcal{L} d^{5} x
$$

where

$$
\mathcal{L}=(-\hat{g})^{\frac{1}{2}} \hat{R}^{J K M N} \hat{R}_{J K M N}
$$

which is quadratic in the $5 \mathrm{D}$ curvature tensor. To reduce the quadratic invariant $\hat{\mathcal{I}}_{q}$ into the actual $4 \mathrm{D}$ spacetime, it is expanded as

$$
\hat{\mathcal{I}}_{q}=\hat{R}^{J K M N} \hat{R}_{J K M N}=\hat{R}^{j k m n} \hat{R}_{j k m n}+4 \hat{R}^{j k m 5} \hat{R}_{j k m 5}+4 \hat{R}^{k 5 m 5} \hat{R}_{k 5 m 5}
$$

and the substitution of (6) into above is sufficient.

We adopt the Palatini approach, where the metric and the connection variations are considered to be independent, thus producing two sets of equations. 
Variations of the action (77) with respect to the connection $\delta \mathcal{L} / \delta \Gamma^{A}{ }_{B C}=$ 0 renders

$$
\hat{D}_{K} \hat{R}_{B M N}^{K}=0
$$

and we interpret it as the field equations of the model. Varying with respect to the $5 \mathrm{D}$ metric

$$
\frac{(-\hat{g})^{-1 / 2}}{2} \frac{\delta \mathcal{L}}{\delta \hat{g}_{A B}} \equiv \hat{T}_{A B}
$$

gives

$$
\hat{T}_{A B}=\hat{R}_{A K M N} \hat{R}_{B}{ }^{K M N}-\frac{1}{4} \hat{g}_{A B} \hat{R}_{J K M N} \hat{R}^{J K M N}
$$

and interpreted as the SE tensor of the model which is symmetric and due to the field equations (10) is covariantly conserved

$$
\hat{D}_{K} \hat{T}_{B}^{K}=0 .
$$

This approach is quite similar to that of the gauge theories whose the field equations are obtained by varying with respect to the gauge potentials, while the SE tensor is obtained through the metric variations of the action. The gauge structure of gravity with an appropriate choice of the gauge group is well established [17, 18, 19], where the Riemann tensor and the connection are behaving like the curvature and the gauge potential, respectively. Therefore, the implementation of the Palatini method on the action (77) can be regarded as a natural extension of a familiar approach to gravity.

\section{The Kaluza-Klein Reduction}

The reduced form of the quadratic invariant in (9) becomes

$$
\begin{aligned}
& \hat{\mathcal{I}}_{q}=R^{j k m n} R_{j k m n}-\frac{3}{2} \varphi^{2} R_{j k m n} F^{j k} F^{m n}+\frac{3}{8} \varphi^{4} F_{j k} F^{j k} F_{m n} F^{m n} \\
& +\frac{5}{8} \varphi^{4} F_{j k} F^{k m} F_{m n} F^{n j}+\varphi^{2}\left(D_{k} F_{m n}\right)\left(D^{k} F^{m n}\right) \\
& +6\left(\varphi_{k} \varphi^{k} F_{m n} F^{m n}+\varphi_{m} \varphi_{n} F^{m k} F_{k}^{n}\right) \\
& +4 \varphi\left(\varphi^{m} F^{k n}+\varphi_{k} F^{m n}\right)\left(D_{k} F_{m n}\right) \\
& +4 \varphi^{-2} D_{m} \varphi_{n} D^{m} \varphi^{n}-2 \varphi D_{m} \varphi_{n} F^{m k} F_{k}^{n},
\end{aligned}
$$

which has also been earlier evaluated in [9] using differential forms, and appears to be different from above due to our contingent use of gauge and gravitational Bianchi identities:

$$
D_{[k} F_{m n]}=0, \quad D_{[k} R_{m n] i j}=0 .
$$


In the sequel, we shall also be using

$$
\left(D_{m} D_{n}-D_{n} D_{m}\right) F_{j}^{i}=R_{k m n}^{i} F_{j}^{k}-R_{j m n}^{k} F_{k}^{i}
$$

and

$$
2 R_{j k m n} F^{j m} F^{k n}=R_{j k m n} F^{j k} F^{m n}
$$

whenever they happen to be useful for our purposes.

\subsection{The Reduction of the Field Equations}

The field equations (10) comprises four equations to be reduced

$\hat{D}_{K} \hat{R}_{b m n}^{K}=0, \quad \hat{D}_{K} \hat{R}^{K}{ }_{5 m n}=0, \quad \hat{D}_{K} \hat{R}_{b 5 n}^{K}=0, \quad \hat{D}_{K} \hat{R}^{K}{ }_{5 m 5}=0$

The first of the above equations becomes

$$
\begin{aligned}
& D_{k} R_{b m n}^{k}+\varphi^{2}\left\{\frac{1}{4}\left[F_{b m} D_{k} F^{k}{ }_{n}-F_{b n} D_{k} F^{k}{ }_{m}\right]\right. \\
& \left.\frac{1}{2}\left[F_{n k} D_{m} F_{b}^{k}-F_{m k} D_{n} F_{b}^{k}-D_{k}\left(F^{k}{ }_{b} F_{m n}\right)\right]\right\} \\
& +\varphi^{-1} \varphi_{k} R^{k}{ }_{b m n} \\
& +\varphi^{-2}\left(\varphi_{m} D_{n} \varphi_{b}-\varphi_{n} D_{m} \varphi_{b}\right) \\
& -\frac{3}{4} \varphi \varphi_{k}\left(2 F^{k}{ }_{b} F_{m n}+F^{k}{ }_{m} F_{b n}-F^{k}{ }_{n} F_{b m}\right) \\
& +\varphi\left(\varphi_{m} F_{b k} F_{n}^{k}-\varphi_{n} F_{b k} F_{m}^{k}\right)=0
\end{aligned}
$$

and the second equation is reduced as

$$
\begin{aligned}
& -\frac{1}{2} \varphi\left(F^{j k} R_{j k m n}+D_{k} D^{k} F_{m n}\right)+\frac{1}{4} \varphi^{3} F^{j k}\left(F_{j k} F_{m n}+2 F_{j m} F_{k n}\right) \\
& +\left(F_{n}{ }^{k} D_{m} \varphi_{k}-F_{m}{ }^{k} D_{n} \varphi_{k}\right) \\
& +\frac{1}{2}\left(\varphi_{n} D_{k} F_{m}^{k}-\varphi_{m} D_{k} F_{n}^{k}\right) \\
& -\frac{3}{2} \varphi^{k} D_{k} F_{m n}-F_{m n} D_{k} \varphi^{k}=0 .
\end{aligned}
$$

The third equation becomes

$$
\begin{aligned}
& \frac{1}{2} \varphi\left(F^{j k} R_{k b j n}+D_{k} D_{n} F_{b}^{k}\right)+\frac{1}{8} \varphi^{3} F^{j k}\left(F_{j k} F_{b n}+2 F_{j b} F_{k n}\right) \\
& -F_{b}{ }^{k} D_{k} \varphi_{n}-\frac{1}{2} F_{b n} D_{k} \varphi^{k} \\
& +\frac{3}{2} \varphi^{-1} \varphi_{k}\left(\varphi_{n} F^{k}{ }_{b}+\varphi_{b} F^{k}{ }_{n}\right) \\
& +\frac{3}{2} \varphi_{k} D_{n} F_{b}^{k}+\varphi_{n} D_{k} F_{b}^{k}+\frac{1}{2} \varphi_{b} D_{k} F_{n}^{k}=0 .
\end{aligned}
$$


Similarly, through the reduction recipe last equation takes the following form

$$
\begin{aligned}
& \frac{1}{4} \varphi^{2}\left[F_{m j} D_{k} F^{k j}+D_{m}\left(F^{j k} F_{j k}\right)\right] \\
& +\varphi^{-2} \varphi^{j} D_{m} \varphi_{j}-\varphi^{-1} D_{j} D^{j} \varphi_{m} \\
& -\frac{5}{4} \varphi \varphi_{j} F^{j k} F_{k m}-\frac{3}{4} \varphi \varphi_{m} F^{j k} F_{k j}=0 .
\end{aligned}
$$

Considering the special case $\varphi=$ constant, the equation above simplifies as:

$$
F_{m j} D_{k} F^{k j}+D_{m}\left(F^{j k} F_{j k}\right)=0 .
$$

Introducing $F^{2}=F^{j k} F_{j k}$ and $J^{j}=D_{k} F^{k j}$, and interpreting the latter as the current density, it can be expressed as

$$
F_{m j} J^{j}=-D_{m} F^{2}
$$

where, it can safely be interpreted as the "Lorentz force", which is derived from a scalar $F^{2}$. Lorentz force within the context of the $5 \mathrm{D}$ geodesic equation has been presented earlier in [20].

We shall reorganize the field equations from (18) to (21) by freely using identities. Then (18) becomes

$$
\begin{aligned}
& D_{n}\left(R_{b m}-\frac{1}{2} \varphi^{2} F_{b k} F_{m}{ }^{k}-\varphi^{-1} D_{m} \varphi_{b}\right) \\
& -D_{m}\left(R_{b n}-\frac{1}{2} \varphi^{2} F_{b k} F_{n}{ }^{k}-\varphi^{-1} D_{n} \varphi_{b}\right) \\
& +\frac{1}{4}\left[F_{b n}\left(D_{k} F^{k}{ }_{m}+3 \varphi^{-1} \varphi_{k} F^{k}{ }_{m}\right)-F_{b m}\left(D_{k} F^{k}{ }_{n}+3 \varphi^{-1} \varphi_{k} F^{k}{ }_{n}\right)\right. \\
& \left.+2 F_{m n}\left(D_{k} F^{k}{ }_{b}+3 \varphi^{-1} \varphi_{k} F^{k}{ }_{b}\right)\right]=0
\end{aligned}
$$

and equation (19) is organized as

$$
\begin{aligned}
& D_{m}\left(D_{k} F_{n}^{k}+3 \varphi^{-1} \varphi_{k} F_{n}^{k}\right)-D_{n}\left(D_{k} F_{m}^{k}+3 \varphi^{-1} \varphi_{k} F_{m}^{k}\right) \\
& +F_{m}{ }^{k}\left(R_{k n}-\frac{1}{2} \varphi^{2} F_{k j} F_{n}{ }^{j}-\varphi^{-1} D_{k} \varphi_{n}\right) \\
& -F_{n}{ }^{k}\left(R_{k m}-\frac{1}{2} \varphi^{2} F_{k j} F_{m}{ }^{j}-\varphi^{-1} D_{k} \varphi_{m}\right) \\
& +2 \varphi^{-1} F_{m n}\left(D_{j} \varphi^{j}-\frac{1}{4} \varphi^{3} F_{j k} F^{j k}\right)=0 .
\end{aligned}
$$

The third equation (20) is similarly rearranged as

$$
\begin{aligned}
& \varphi D_{n}\left(D_{k} F_{b}^{k}+3 \varphi^{-1} \varphi_{k} F_{b}^{k}\right) \\
& +\varphi_{b}\left(D_{k} F_{n}^{k}+3 \varphi^{-1} \varphi_{k} F^{k}{ }_{n}\right)+2 \varphi_{n}\left(D_{k} F^{k}{ }_{b}+3 \varphi^{-1} \varphi_{k} F^{k}{ }_{b}\right) \\
& +F_{n b}\left(D_{k} \varphi^{k}-\frac{1}{4} \varphi^{3} F_{j k} F^{j k}\right) \\
& -\varphi F_{b}{ }^{k}\left(R_{k n}-\frac{1}{2} \varphi^{2} F_{k j} F_{n}{ }^{j}-\varphi^{-1} D_{k} \varphi_{n}\right)=0
\end{aligned}
$$


and the last equation (21) takes the form

$$
\begin{aligned}
& D_{m}\left(D_{k} \varphi^{k}-\frac{1}{4} \varphi^{3} F_{j k} F^{j k}\right) \\
& -\frac{1}{4} \varphi^{3} F_{m}{ }^{j}\left(D_{k} F^{k}{ }_{j}+3 \varphi^{-1} \varphi_{k} F^{k}{ }_{j}\right) \\
& +\varphi_{j}\left(R_{j m}-\frac{1}{2} \varphi^{2} F_{j k} F_{m}{ }^{k}-\varphi^{-1} D_{j} \varphi_{m}\right)=0 .
\end{aligned}
$$

Looking closely into the above four equations, it is now possible to recognize the patterns of the SKK equations in (5). Equation (24) contains the covariant derivative of $K 1_{a b}$ and particular couplings of $K 2_{a}$ and $K 3$ with the EM field strength tensor. Similarly, (25) and (26) govern $F_{m n}$ containing the covariant derivative of $K 2_{a}$ and have couplings of $F_{m n}$ and $\varphi$ with the whole $K$-set of (5). The last equation (27), includes the covariant derivative of $K 3$, basically governs the dilaton field and has couplings of $K 2_{a}$ and $K 1_{a b}$ with $F_{m n}$ and $\varphi$. It can be seen that neither $R_{j k m n}$ nor $R_{a b}$ has any couplings with the $K$-set. Therefore, any solution to the SKK equations also solves the field equations (24)-(27).

\subsection{The Reduction of the SE-Tensor}

The $\hat{T}_{a b}$ component of the reduced stress-energy tensor in (12) can be separated into its trace-free and a non-vanishing trace part. Its trace-free part is:

$$
\begin{aligned}
\hat{T}_{a b}^{(\text {tracefree })=} & R_{a k m n} R_{b}{ }^{k m n}-\frac{1}{4} g_{a b} R_{j k m n} R^{j k m n} \\
& -\frac{3}{2} \varphi^{2} F^{m n}\left\{\frac{1}{2}\left(F_{a}{ }^{k} R_{b k m n}+F_{b}{ }^{k} R_{a k m n}\right)-\frac{1}{4} g_{a b} F^{j k} R_{j k m n}\right\} \\
& +\frac{3}{8} \varphi^{4} F^{2}\left(F_{a k} F_{b}{ }^{k}-\frac{1}{4} g_{a b} F_{j k} F^{j k}\right) \\
& +\frac{5}{8} \varphi^{4}\left(F_{a m} F^{m k} F_{k n} F^{n}{ }_{b}-\frac{1}{4} g_{a b}\left(F_{j k} F^{k m} F_{m n} F^{n j}\right)\right) \\
& +\varphi^{2}\left\{\left(D_{a} F_{m n}\right)\left(D_{b} F^{m n}\right)-\frac{1}{4} g_{a b}\left(D_{k} F_{m n}\right)\left(D^{k} F^{m n}\right)\right\} \\
& +4 \varphi^{-2}\left\{\left(D_{a} \varphi_{m}\right)\left(D_{b} \varphi^{m}\right)-\frac{1}{4} g_{a b}\left(D_{n} \varphi_{m}\right)\left(D^{n} \varphi^{m}\right)\right\} \\
& +3 \varphi_{m} \varphi^{m}\left(F_{a k} F_{b}{ }^{k}-\frac{1}{4} g_{a b} F_{j k} F^{j k}\right) \\
& +3 F^{2}\left(\varphi_{a} \varphi_{b}-\frac{1}{4} g_{a b} \varphi_{k} \varphi^{k}\right) \\
& +3 \varphi^{j} \varphi^{k}\left(F_{a j} F_{b k}-\frac{1}{4} g_{a b} F_{n j} F_{k}^{n}\right)
\end{aligned}
$$




$$
\begin{aligned}
& +3\left\{\varphi_{k} F^{k j}\left[\frac{1}{2}\left(\varphi_{a} F_{b j}+\varphi_{b} F_{a j}\right)-\frac{1}{4} g_{a b} \varphi_{m} F^{m}{ }_{j}\right]\right\} \\
& -4 \varphi\left\{F^{j k}\left[\frac{1}{2}\left(\varphi_{a} D_{j} F_{k b}+\varphi_{b} D_{j} F_{k a}\right)-\frac{1}{4} g_{a b} \varphi^{n} D_{j} F_{k n}\right]\right\} \\
& +4 \varphi\left\{\varphi^{k}\left[\frac{1}{2}\left(F_{a}{ }^{n} D_{k} F_{b n}+F_{b}{ }^{n} D_{k} F_{a n}\right)-\frac{1}{4} g_{a b} F^{j k} D_{m} F_{j k}\right]\right\} \\
& +2 \varphi\left\{\frac{1}{2}\left[\left(D_{a} \varphi_{m}\right) F_{b j} F^{j m}+\left(D_{b} \varphi_{m}\right) F_{a j} F^{j m}\right]-\frac{1}{4} g_{a b}\left(D_{n} \varphi_{m}\right) F^{n k} F_{k}{ }^{m}\right\}
\end{aligned}
$$

and the part with a non-vanishing trace is:

$$
\begin{aligned}
\hat{T}_{a b}^{(t a)}= & -\frac{1}{8} \varphi^{4} F_{a m} F^{m k} F_{k n} F_{b}^{n}+\frac{1}{2} \varphi^{2}\left(D_{m} F_{a k}\right)\left(D^{m} F_{b}{ }^{k}\right)-\frac{3}{4} \varphi^{2}\left(D_{a} F_{m n}\right)\left(D_{b} F^{m n}\right) \\
& -2 \varphi^{-2}\left(D_{a} \varphi_{m}\right)\left(D_{b} \varphi^{m}\right)-\frac{3}{2} F^{2} \varphi_{a} \varphi_{b}-\frac{3}{2} \varphi_{j} \varphi_{k} F_{a}{ }^{j} F_{b}{ }^{k} \\
& -\frac{1}{2} \varphi F^{j m}\left(F_{a j} D_{b} \varphi_{m}+F_{b j} D_{a} \varphi_{m}\right)+\frac{1}{2} \varphi\left(\varphi_{a} D_{m} F_{n b}+\varphi_{b} D^{m} F_{n a}\right) \\
& -\frac{1}{2} \varphi \varphi^{k} F_{a k}\left(D_{m} F_{b}{ }^{k}+F_{b}{ }^{k} D_{m} F_{a k}\right) .
\end{aligned}
$$

The reduced $\hat{T}_{a 5}$ component of the SE tensor can be written as:

$$
\begin{aligned}
\hat{T}_{a 5}= & 3 \varphi^{-1} F_{a}^{m} \varphi^{k} D_{m} \varphi_{k} \\
& +\left(D_{m} \varphi_{k}\right) D^{m} F^{k}{ }_{a}+R_{a k m n}\left(\varphi^{k} F^{m n}+\varphi^{m} F^{k n}\right) \\
& +\frac{1}{2} \varphi R_{a k m n} D^{k} F^{m n}+\frac{3}{4} \varphi^{2} F^{2} \varphi_{k} F^{k}{ }_{a} \\
& +\frac{1}{4} \varphi^{3}\left(3 F_{a}{ }^{k} F^{m n} D_{m} F_{n k}+F^{k}{ }_{n} F^{n m} D_{m} F_{k a}\right) .
\end{aligned}
$$

And the last component $\hat{T}_{55}$ is reduced in the following manner:

$$
\begin{aligned}
\hat{T}_{55}= & \varphi^{-2}\left(D_{m} \varphi_{n}\right) D^{m} \varphi^{n}-\frac{1}{4} R_{j k m n} R^{j k m n}+\frac{1}{2} \varphi F^{m}{ }_{n} F^{n k} D_{k} \varphi_{m} \\
& +\frac{3}{8} \varphi^{2} R_{j k m n} F^{j k} F^{m n}-\frac{1}{32} \varphi^{4}\left(3 F^{4}+F_{j k} F^{k m} F_{m n} F^{n j}\right) .
\end{aligned}
$$

The trace of the $\hat{T}_{A B}$ can be written as

$$
\hat{T}=\hat{g}^{A B} T_{A B}=\hat{T}_{a}^{a}+\hat{T}^{5}{ }_{5}=-\frac{1}{4} \hat{\mathcal{I}}_{q} .
$$

Therefore, the SE tensor is trace-free only when the invariant $\hat{\mathcal{I}}_{q}$ vanishes. 


\section{Conclusion}

As one would expect the dimensionally reduced $4 \mathrm{D}$ field equations are more involved than those of the usual Kilmister-Yang (KY) type of gravity, containing non-minimal couplings of Ricci tensor and the field tensor of EM along with a scalar dilaton field. We have explicitly shown that our field equations are some specific combinations of the equations of the SKK theory.

It is found out that the set of field equations also contain the Lorentz force in addition to some particular couplings with Maxwell's equations. As is well known, the theory of EM is only complete when the Lorentz force is also taken into account.

The stress energy tensor contains particular non-minimal couplings with the well known SE tensors of the KY type of gravity, EM and the dilaton field. Although they appear to be more complicated than the standard SE tensors of those fields when considered separately, it seems to be the price we have to pay for unification and thus for mutual interactions. We have also analyzed the trace of the SE tensor and its conservation properties and provided the conditions for trace-freedom.

It is known that the KY pure gravity equations contain non-physical solutions. In dimensions higher than four, the introduction of the GaussBonnet action seemed to provide some relief. In the context of the KK theory, couplings with the EM and the dilaton field requires more equations governing the fields that may alleviate the problem. A through analysis on this matter and on the existence of any further possible remedies is left to a later study.

Finally we conclude that this model turned out to be more complete by accommodating the Lorentz force and an intrinsic inclusion of the solutions of the SKK model[21].

\section{References}

[1] Kaluza Th 1921 Sitz. Preuss. Acad. Wiss. Phys. Math. K1 966

[2] Duff M J hep-th/9410046

[3] Canfora F, Giacomini A and Zerwekh A R 2009 Phys. Rev. D 80084039

[4] Klein O 1926 Z. Phys. 37895

[5] Allahverdizadeh M, Matsuno K and Sheykhi A 2010 Phys. Rev. D 81 044001 
[6] Müller-Hoissen F 1988 Class. Quantum Grav. 3665

[7] Müller-Hoissen F 1988 Phys. Lett. B 201325

[8] Huang W H 1988 Phys. Lett. B 203105

[9] Dereli T and Uçoluk G 1990 Class. Quantum Grav. 71109

[10] Soleng H H and Gron O 1995 Annals Phys. 240432

[11] Sotiriou T P and Faraoni V 2010 Rev. Mod. Phys 82451

[12] Boulware D G and Deser S 1985 Phys. Rev. Lett. 552656

[13] Stelle K S 1977 Phys. Rev. D 16953

[14] Macias A and Dehnen H 1991 Class. Quantum Grav. 8203

[15] Misner C W, Thorne K S and Wheeler J A 1973 Gravitation (W. H. Freeman, San Francisco) Chap 8

[16] Wesson P S 1999 Space, Time, Matter: Modern Kaluza-Klein Theory (World Scientific Publishing Co. Pte. Ltd.) p 149

[17] Yang C N 1974 Phys. Rev. Lett. 33445

[18] Mansouri F and Chang L N 1976 Phys. Rev. D 133192

[19] Gronwald F and Hehl F W gr-qc/9602013

[20] Kerner R, Martin J, Mignemi S and van Holten J W 2000 Phys. Rev. D 63027502

[21] Kuyrukcu H 2010 Kaluza-Klein Reduction of Higher Curvature Gravity Models (PhD Thesis, Middle East Technical University) 